ANNALES

POLONICI MATHEMATICI

LXXVII.3 (2001)

\title{
Universality of derivative and antiderivative operators with holomorphic coefficients
}

\author{
by María Del Carmen Calderón-Moreno (Sevilla)
}

\begin{abstract}
We prove some conditions on a sequence of functions and on a complex domain for the existence of universal functions with respect to sequences of certain derivative and antiderivative operators related to them. Conditions for the equicontinuity of those families of operators are also studied. The conditions depend upon the "size" of the domain and functions. Some earlier results about multiplicative complex sequences are extended.
\end{abstract}

1. Introduction and notation. In this paper, we denote by $\mathbb{C}$ the complex plane and by $G$ a simply connected domain, i.e., $G \subset \mathbb{C}, G$ is nonempty, open and connected, and its complement with respect to the extended plane is also connected. $H(G)$ denotes, as usual, the space of holomorphic functions in $G$, endowed with the topology of uniform convergence on compact subsets.

$B(a, r)$ (resp. $\bar{B}(a, r))$ is the euclidean open (resp. closed) disk with center $a$ and radius $r$. We agree that $B(a,+\infty)=\mathbb{C}$. $\mathbb{N}$ is the set of positive integers. We define $\mathcal{K}(G)=\{K \subset G: K$ is compact $\}$ and $\|g\|_{A}=\sup _{z \in A}|g(z)|$, where $g$ is a complex function defined on a subset $A \subset \mathbb{C}$. If $A, B \subset \mathbb{C}$ then $d(A, B)=\inf \{|a-b|: a \in A, b \in B\}$ and $\operatorname{diam}(A)=\sup \{|a-b|: a, b \in A\}$. We adopt the conventions $d(a, \emptyset)=+\infty(a \in \mathbb{C}), 1 /+\infty=0$ and $1 / 0=+\infty$. The circumscribed radius of $G$ is defined as

$$
\begin{aligned}
R(G) & =\inf _{a \in \mathbb{C}} \sup _{b \in G}|a-b| \\
& =\inf \{r>0: \text { there is an open disk } B \text { of radius } r \text { with } G \subset B\}
\end{aligned}
$$

and the inscribed radius of $G$ as

2000 Mathematics Subject Classification: Primary 30E10; Secondary 47B38, 47E05.

Key words and phrases: universal function, residual set, derivative operator, antiderivative operator, size and shape of a domain.

This work is supported in part by DGES grant PB96-1348 and the Junta de Andalucía. 


$$
\begin{aligned}
\varrho(G) & =\sup _{b \in G} \inf _{a \notin G}|a-b| \\
& =\sup \{r>0: \text { there is an open disk } B \text { of radius } r \text { with } B \subset G\} .
\end{aligned}
$$

The numbers $\operatorname{diam}(G), R(G)$ and $\varrho(G)$ describe, elementarily, the "size" of the domain $G$. A point $a \in G$ is an interior center of $G$ when $B(a, \varrho(G)) \subset G$. We denote by $\operatorname{ct}(G)$ the set of all interior centers of $G$. In a similar way, $a \in \mathbb{C}$ is an exterior center of $G$ if $G \subset B(a, R(G))$, and $\mathrm{CT}(G)$ denotes the set of all exterior centers of $G$.

If $a \in G$, then we set

$\Delta_{a}(G)=\sup _{z \in G} \inf \{r>0: a$ is in the connected component of $B(z, r) \cap G$ containing $z\}$

(finite or $+\infty$ ). Finally, we denote by $\Delta(G)$ the number (finite or not)

$$
\Delta(G)=\inf _{a \in G} \Delta_{a}(G) .
$$

Observe that if $\Delta_{a}(G)$ is finite then it is the smallest $\delta>0$ such that for any $z \in G$ there is a rectifiable arc $\gamma \subset G$ joining $a$ to $z$ with $|t-z|<\delta$ for all $t \in \gamma$. If $\Delta(G)$ is finite then it is the smallest number $\delta>0$ with the following property:

(P) Given $\varepsilon>0$, there is $a=a(\varepsilon) \in G$ such that for all $z \in G$ there is a rectifiable $\operatorname{arc} \gamma \subset G$ joining $a$ to $z$ with $|t-z|<\delta+\varepsilon$ for all $t \in \gamma$.

The next proposition has a geometric character and it is interesting in itself. It will not be used in its totality in the remaining results. In it we put together some "size" and "shape" properties of a domain. The proof will not be given, but a few pictures may help the reader to visualize all the properties.

Proposition 1.1. With the previous notations and conventions, the following properties are satisfied:

(1) $0<\varrho(G) \leq \frac{1}{2} \operatorname{diam}(G) \leq R(G) \leq \frac{\sqrt{3}}{2} \operatorname{diam}(G)$.

(2) $R(G) \leq \Delta(G) \leq \Delta_{a}(G) \leq \operatorname{diam}(G)$ for all $a \in G$.

(3) The following conditions are equivalent:

(a) $G$ is bounded.

(b) $\operatorname{diam}(G)<+\infty$.

(c) $R(G)<+\infty$.

(d) $\Delta(G)<+\infty$.

(e) $\mathrm{CT}(G)$ has only one point.

(f) $\mathrm{CT}(G) \neq \mathbb{C}$.

(4) If $G$ is bounded, then $\varrho(G)=R(G)$ if and only if $G$ is an open disk.

(5) $d(a, \mathbb{C} \backslash G) \leq \sup _{z \in G}|z-a| \leq \Delta_{a}(G)$ for all $a \in G$.

(6) If $G$ is starlike with respect to a then $\Delta(G)=\sup _{z \in G}|z-a|$. 
(7) $d(a, \mathbb{C} \backslash G)=\Delta_{a}(G)$ if and only if $G=\mathbb{C}$ or $G$ is an open disk with center $a$. In that case, $d(a, \mathbb{C} \backslash G)=\Delta_{a}(G)=$ radius of $G$.

(8) If $\varrho(G)=+\infty$, then $\operatorname{ct}(G) \neq \emptyset$ if and only if $G=\mathbb{C}$.

(9) If $R(G)=\Delta(G)$ then $d(\mathrm{CT}(G), G)=0$.

(10) If $\mathrm{CT}(G) \cap G=\emptyset$, then $\Delta(G)<\Delta_{a}(G)$ for all $a \in G$.

(11) If $\lim _{n \rightarrow \infty} a_{n}=a$, then $\lim _{n \rightarrow \infty} \Delta_{a_{n}}(G)=\Delta_{a}(G)$.

(12) There is a $\in G$ such that $\Delta(G)=\Delta_{a}(G)$ if and only if either $G$ is bounded and $\mathrm{CT}(G) \subset G$ or $G$ is not bounded.

REMARK 1.2. There are domains where the above parameters satisfy

$$
\varrho(G)<R(G)<\Delta(G)<\operatorname{diam}(G) .
$$

EXAMPLE 1.3. We consider the "horseshoe"

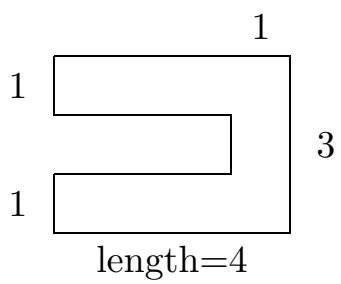

If $G$ is its interior, then

$$
\frac{1}{2}<\varrho(G)<1<\frac{5}{2}=R(G)<\sqrt{13}=\Delta(G)<5=\operatorname{diam}(G) .
$$

The set of polynomials is dense in $H(G)$ by Runge's theorem [11]. $H(G)$ is a second-countable Fréchet space. By Baire's Category Theorem, $H(G)$ is a Baire space, i.e., the intersection of a countable family of open dense subsets is also dense. In a Baire space $X$, a subset is residual when it contains a dense $G_{\delta}$-subset of $X$. Such a subset is "very large" in $X$. For this see, for instance, [7, pp. 213-214 and 238] and [10, pp. 40-41].

The following general notion of universality can be found in [6]: Let $X$ and $Y$ be topological spaces and $T_{n}: X \rightarrow Y(n \in \mathbb{N})$ a sequence of continuous mappings. Then an element $x \in X$ is called $\left\{T_{n}\right\}_{n=1}^{\infty}$-universal if its orbit $\left\{T_{n} x: n \in \mathbb{N}\right\}$ is dense in $Y$. Several versions of the following universality criterion can be found in [5].

TheOREM 1.4. Let $X$ be a linear topological space that is a Baire space, $Y$ a linear topological space that is second-countable, $D \subset X$ dense in $X$, $D^{\prime} \subset Y$ dense in $Y$ and $T_{n}: X \rightarrow Y(n \in \mathbb{N})$ a countable family of continuous linear mappings satisfying the following condition:

(A) For every $d \in D$ and every $d^{\prime} \in D^{\prime}$ there exists a sequence $\left\{x_{p}\right.$ : $p \in \mathbb{N}\} \subset X$ and positive integers $n_{1}<n_{2}<\ldots$ such that $x_{p} \rightarrow 0$, $T_{n_{p}}(d) \rightarrow 0$ and $T_{n_{p}}\left(x_{p}\right) \rightarrow d^{\prime}(p \rightarrow \infty)$.

Then the subset of $\left\{T_{n}\right\}_{n=1}^{\infty}$-universal vectors $x \in X$ is residual in $X$. 
We point out that if $X$ and $Y$ are two nontrivial metric linear spaces and $\left\{T_{n}: n \in \mathbb{N}\right\}$ is an equicontinuous family of linear operators from $X$ into $Y$, then the orbit $\left\{T_{n} x: n \in \mathbb{N}\right\}$ of each $x \in X$ is bounded; so there is no $\left\{T_{n}\right\}_{n=1}^{\infty}$-universal vector $x$.

In this paper the derivative and antiderivative operators will be considered. These are defined as follows. The derivative operators are

$$
D^{n}: H(G) \ni f \mapsto f^{(n)} \in H(G) \quad(n \in \mathbb{N}) .
$$

If a point $a \in G$ is fixed, the antiderivative operators are

$$
I^{n}: H(G) \ni f \mapsto \int_{a}^{z} I^{n-1} f(t) d t \in H(G) \quad(n \in \mathbb{N}),
$$

with $I^{0} f=f$, the integral being taken along any rectifiable arc in $G$ joining $a$ to $z$. These operators are linear and continuous. Further, we have

$$
I^{n} f(z)=\frac{1}{(n-1) !} \int_{a}^{z}(z-t)^{n-1} f(t) d t \quad(\forall n \in \mathbb{N}, \forall f \in H(G), \forall z \in G) .
$$

Along the last few decades the universality of the family of operators $\left\{D^{n}\right\}_{n=1}^{\infty}$ has been extensively studied. MacLane [9] stated that there exist $\left\{D^{n}\right\}$-universal vectors in $H(\mathbb{C})$ and Duios Ruis [3] proved that there is a residual set of such functions. Furthermore Gethner and Shapiro [4] and Grosse-Erdmann [6, Satz 2.2.8] got the same result for every simply connected domain. Trivially, there cannot be any $\left\{I_{n}\right\}_{n=1}^{\infty}$-universal function $f \in H(G)$ because $I^{n} f(a)=0$ for $n \in \mathbb{N}$. Nevertheless, it is shown in [2] that there exists a sequence $\left\{C_{n}\right\}_{n=1}^{\infty} \subset \mathbb{C}$ with the following property: For every entire function $\Phi$ the set $\left\{Q_{n}(z)=I^{n} \Phi(z)+\sum_{j=0}^{n-1}\left(C_{n-j} / j !\right) z^{j}: n \in \mathbb{N}\right\}$ is dense in $H(\mathbb{C})$. Luh [8] extended this to functions $\Phi$ which are holomorphic in an open set with simply connected components. L. Bernal-González [1] generalized these results by taking into account the operators $c_{n} D^{n}, c_{n} I^{n}$, where $\left\{c_{n}\right\}_{n=1}^{\infty}$ is a sequence in $\mathbb{C}$. Equicontinuity is also studied in [1].

All these results will in turn be extended in this paper, by inserting this time any sequence $\left\{c_{n}(z)\right\}_{n=1}^{\infty}$ of functions in $H(G)$. As in [1], the "size" of the domain $G$ besides the "size" of $\left\{c_{n}(z)\right\}_{n=1}^{\infty}$ will play an important role. As can be expected, the formulation of the corresponding properties is more involved.

With this aim, let $\left\{c_{n}(z)\right\}_{n=1}^{\infty}$ be a sequence of functions in $H(G)$. We will study the universality and equicontinuity of the families of operators

$$
c_{n}(\cdot) D^{n}: H(G) \ni f \mapsto c_{n}(\cdot) f^{(n)} \in H(G)
$$

and

$$
c_{n}(\cdot) I^{n}: H(G) \ni f \mapsto c_{n}(\cdot) I^{n} f \in H(G) .
$$

Each of these operators is obviously linear and continuous. 
We associate with the functions $c_{n}(z)$ certain numbers in $[0,+\infty]$. If $K \in \mathcal{K}(G)$ and $b \in G$ then we define

$$
\begin{aligned}
& \alpha_{1}(K)=\limsup _{n \rightarrow \infty}\left(n ! \sup _{z \in K}\left|c_{n}(z)\right|\right)^{1 / n}=\frac{1}{e} \limsup _{n \rightarrow \infty}\left(n \sup _{z \in K}\left|c_{n}(z)\right|^{1 / n}\right), \\
& \alpha_{2}(K)=\limsup _{n \rightarrow \infty}\left(n ! \inf _{z \in K}\left|c_{n}(z)\right|\right)^{1 / n}=\frac{1}{e} \limsup _{n \rightarrow \infty}\left(n \inf _{z \in K}\left|c_{n}(z)\right|^{\frac{1}{n}}\right), \\
& \alpha_{3}(b)=\limsup _{n \rightarrow \infty}\left(n !\left|c_{n}(b)\right|\right)^{1 / n}=\frac{1}{e} \limsup _{n \rightarrow \infty}\left(n\left|c_{n}(b)\right|^{1 / n}\right), \\
& \beta_{1}(K)=\limsup _{n \rightarrow \infty}\left(\frac{\sup _{z \in K}\left|c_{n}(z)\right|}{n !}\right)^{1 / n}=e \limsup _{n \rightarrow \infty}\left(\frac{\sup _{z \in K}\left|c_{n}(z)\right|^{1 / n}}{n}\right), \\
& \beta_{2}(K)=\limsup _{n \rightarrow \infty}\left(\frac{\inf _{z \in K}\left|c_{n}(z)\right|}{n !}\right)^{1 / n}=e \limsup _{n \rightarrow \infty}\left(\frac{\inf _{z \in K}\left|c_{n}(z)\right|^{1 / n}}{n}\right) .
\end{aligned}
$$

The equalities hold by Stirling's formula. Finally, we define

$$
\begin{gathered}
A_{1}=\sup _{K \in \mathcal{K}(G)} \alpha_{1}(K), \quad A_{2}=\inf _{K \in \mathcal{K}(G)} \alpha_{2}(K), \quad A_{3}=\sup _{b \in G} \alpha_{3}(b), \\
B_{1}=\sup _{K \in \mathcal{K}(G)} \beta_{1}(K), \quad B_{2}=\inf _{K \in \mathcal{K}(G)} \beta_{2}(K) .
\end{gathered}
$$

REMARK 1.5. It is easy to check the following: If $\left\{K_{j}\right\}_{j=1}^{\infty}$ is an exhaustive sequence of compact sets in $G$, i.e., $K_{j} \in \mathcal{K}(G), K_{j} \subset K_{j+1}^{0}$ for all $j \in \mathbb{N}$ and $\bigcup_{j=1}^{\infty} K_{j}=G$, then

$$
\begin{aligned}
& A_{1}=\sup _{j \in \mathbb{N}} \alpha_{1}\left(K_{j}\right)=\lim _{j \rightarrow \infty} \alpha_{1}\left(K_{j}\right), \\
& A_{2}=\inf _{j \in \mathbb{N}} \alpha_{2}\left(K_{j}\right)=\lim _{j \rightarrow \infty} \alpha_{2}\left(K_{j}\right),
\end{aligned}
$$

and analogously for $B_{1}$ and $B_{2}$.

REMARK 1.6. In the particular case that $c_{n}(z) \equiv c_{n}$ is a constant for each $n \in \mathbb{N}$, we have

$$
A_{1}=A_{2}=A_{3}=\limsup _{n \rightarrow \infty}\left(n !\left|c_{n}\right|\right)^{1 / n}(=\alpha)
$$

and

$$
B_{1}=B_{2}=\limsup _{n \rightarrow \infty}\left(\left|c_{n}\right| / n !\right)^{1 / n}(=\beta) .
$$

The numbers $\alpha, \beta$ were introduced in [1].

REMARK 1.7. In general, we can only say that

$$
A_{2} \leq A_{3} \leq A_{1} \quad \text { and } \quad B_{2} \leq B_{1} .
$$

2. Derivative operators. First, we ask if there exists $f \in H(G)$ such that the orbit $\left\{c_{n}(\cdot) f^{(n)}: n \in \mathbb{N}\right\}$ is dense. The next result furnishes a sufficient condition. 
Theorem 2.1. If $\Delta(G)<A_{2}$ and $c_{n}(z) \neq 0$ for all $z \in G$ and $n \in \mathbb{N}$, then the subset of $f \in H(G)$ such that the orbit $\left\{c_{n}(\cdot) f^{(n)}: n \in \mathbb{N}\right\}$ is dense in $H(G)$ is residual.

Proof. Apply Theorem 1.4 to $X=Y=H(G), D=D^{\prime}=$ \{polynomials and $T_{n}=c_{n}(\cdot) D^{n}(n \in \mathbb{N})$. Fix two polynomials $P, Q$. Trivially, $T_{n}(P)=0$ for all $n>\operatorname{deg}(P)$. Choose $a \in G$ such that $\Delta_{a}(G)<A_{2}$. Then $Q(z)=$ $\sum_{j=0}^{m} a_{j}(z-a)^{j}$ for suitable complex constants $a_{0}, a_{1}, \ldots, a_{m}$. Consider the functions $f_{n}$ defined by

$$
\begin{aligned}
f_{n}(z) & =\frac{1}{(n-1) !} \int_{a}^{z} \frac{(z-t)^{n-1} Q(t)}{c_{n}(t)} d t \\
& =\frac{1}{(n-1) !} \sum_{j=0}^{m} a_{j} \int_{a}^{z} \frac{(z-t)^{n-1}(t-a)^{j}}{c_{n}(t)} d t
\end{aligned}
$$

where the integral is taken along any rectifiable $\operatorname{arc}$ in $G$ joining $a$ to $z$. These functions are well defined because $c_{n}(t) \neq 0$ for all $t \in G$ and $n \in \mathbb{N}$, and $G$ is simply connected. Also, $f_{n} \in H(G)$ and $D^{n} f_{n}(z)=Q(z) / c_{n}(z)$ for all $n \in \mathbb{N}$ and $z \in G$, so $T_{n}\left(f_{n}\right)=Q \rightarrow Q(n \rightarrow \infty)$ in $H(G)$.

Then, by Theorem 1.4, it is sufficient to find a subsequence $\left\{f_{n_{p}}\right\}_{p=1}^{\infty}$ of $\left\{f_{n}\right\}_{n=1}^{\infty}$ such that $f_{n_{p}} \rightarrow 0$ in $H(G)(p \rightarrow \infty)$. If we fix $z \in G$ then, by the definition of $\Delta_{a}(G), a$ is in the connected component of $B\left(z, \Delta_{a}(G) \cup G\right)$ containing $z$. Therefore there is a rectifiable arc $\gamma_{z} \subset G$ joining $a$ to $z$ with $|z-t|<\Delta_{a}(G)$ for all $t \in \gamma_{z}$.

Fix $K \in \mathcal{K}(G)$. We can get a compact set $L$ so that $\bigcup_{z \in K} \gamma_{z} \subset L \subset G$ and $\sup _{z \in K} \operatorname{length}\left(\gamma_{z}\right)=S<\infty$. If $z \in K$ and $n \in \mathbb{N}$, we have

$$
\begin{aligned}
\left|f_{n}(z)\right| & \leq \frac{1}{(n-1) !} \sum_{j=0}^{m}\left|a_{j}\right| \int_{\gamma_{z}} \frac{|z-t|^{n-1}|t-a|^{j}}{\left|c_{n}(t)\right|} d t \\
& \leq \frac{C \Delta_{a}(G)^{n}}{(n-1) ! \inf _{t \in L}\left|c_{n}(t)\right|},
\end{aligned}
$$

where

$$
C:=\frac{1}{\Delta_{a}(G)} \sum_{j=0}^{m}\left|a_{j}\right| S^{j+1} \in(0,+\infty) .
$$

Because $\Delta_{a}(G)<A_{2}$ and $L \in \mathcal{K}(G)$, we have $\Delta_{a}(G)<\alpha_{2}(L)$, and then there exist $\nu>0$ and a sequence $n_{1}<n_{2}<\ldots$ of positive integers with $\Delta_{a}(G)<\nu<\left(\left(n_{p}-1\right) ! \inf _{t \in L}\left|c_{n_{p}}(t)\right|\right)^{1 / n_{p}}$ for all $p \in \mathbb{N}$, so

$$
\frac{\Delta_{a}(G)^{n_{p}}}{\left(n_{p}-1\right) ! \inf _{t \in L}\left|c_{n_{p}}(t)\right|} \leq\left(\frac{\Delta_{a}(G)}{\nu}\right)^{n_{p}} \rightarrow 0 \quad(p \rightarrow \infty)
$$

and, by (1), $f_{n_{p}} \rightarrow 0(p \rightarrow \infty)$ uniformly on $K$. So the theorem is proved. 
Now, we give a necessary condition for the existence of $\left\{c_{n}(\cdot) D^{n}\right\}_{1}^{\infty}$ universal functions. We recall that $\operatorname{ct}(G) \neq \emptyset$ and $\varrho(G)$ is infinite if and only if $G=\mathbb{C}$ (Proposition 1.1(8)). In this case, $\operatorname{ct}(G)=\mathbb{C}$. For the next two theorems we can drop the hypothesis of simple connectedness of $G$.

TheOrem 2.2. Assume that there is a function $f \in H(G)$ whose orbit $\left\{c_{n}(\cdot) f^{(n)}: n \in \mathbb{N}\right\}$ is dense in $H(G)$. Then:

(a) If $\operatorname{ct}(G) \neq \emptyset$ and $\varrho(G)<+\infty$, then $\varrho(G) \leq \inf _{b \in \operatorname{ct}(G)} \alpha_{3}(b)$.

(b) If $G=\mathbb{C}$, then $\alpha_{3}(b)=+\infty$ for all $b \in \mathbb{C}$.

(c) If $\operatorname{ct}(G)=\emptyset$, then $\varrho(G) \leq A_{3}$.

Proof. (a) Assume that $\inf _{b \in \operatorname{ct}(G)} \alpha_{3}(b)<\varrho(G)$. So there is a center $b \in \operatorname{ct}(G)$ such that $\alpha_{3}(b)<\varrho(G)$. Fix $r \in\left(\alpha_{3}(b), \varrho(G)\right)$. By assumption $\bar{B}(b, r) \subset G, \lim _{n \rightarrow \infty} n !\left|c_{n}(b)\right| / r^{n}=0$ and, in view of Cauchy's inequalities,

$$
\left|c_{n}(b) f^{(n)}(b)\right| \leq\left|c_{n}(b)\right| \cdot \frac{n !\|f\|_{\bar{B}(b, r)}}{r^{n}} \rightarrow 0 \quad(n \rightarrow \infty) .
$$

Thus the sequence $\left\{c_{n}(b) f^{(n)}(b)\right\}_{n=1}^{\infty}$ is bounded, and so $\left\{c_{n}(\cdot) f^{(n)}\right\}_{n=1}^{\infty}$ cannot aproximate the constant function $g(z)=1+\sup _{n \in \mathbb{N}}\left|c_{n}(b) f^{(n)}(b)\right|$ on the compact set $K=\{b\}$. This is a contradiction and (a) is proved.

(b) Again assume that $\alpha_{3}(b)<+\infty$ for some $b \in \mathbb{C}$. Let $r \in\left(\alpha_{3}(b),+\infty\right)$ be. Then $\lim _{n \rightarrow \infty} n !\left|c_{n}(b)\right| / r^{n}=0$ and an argument similar to that for (a) leads to a contradiction.

(c) Suppose that $A_{3}<\varrho(G)$. Then there are $b \in G$ and $r \in\left(A_{3}, \varrho(G)\right)$ such that $\bar{B}(b, r) \subset G$. So, $\lim _{\sup _{n \rightarrow \infty}}\left(n !\left|c_{n}(b)\right|\right)^{1 / n}<r$ and just as in (a) and (b) we have a contradiction.

The following two results furnish necessary and sufficient conditions for the equicontinuity. We distinguish the cases $G \neq \mathbb{C}, G=\mathbb{C}$.

TheOREm 2.3. Let $G \subsetneq \mathbb{C}$ be a domain and let $\mathcal{F}$ be the family of operators $\left\{c_{n}(\cdot) D^{n}: n \in \mathbb{N}\right\}$. We have:

(a) If $A_{1}=0$, then $\mathcal{F}$ is equicontinuous.

(b) If $\mathcal{F}$ is equicontinuous, then $A_{2}=0$.

Proof. (a) Fix a basic neighborhood of the origin of $H(G)$, i.e., $V(\varepsilon, K)=$ $\{f \in H(G):|f(z)|<\varepsilon \forall z \in K\}$, where $\varepsilon>0$ and $K \in \mathcal{K}(G)$. We must find $\delta>0$ and $L \in \mathcal{K}(G)$ satisfying

$$
\bigcup_{n \in \mathbb{N}}\left(c_{n}(\cdot) D^{n}\right)(V(\delta, L)) \subset V(\varepsilon, K) .
$$

Pick a cycle $\gamma \subset G \backslash K$ (see [Rud, p. 287]) such that Cauchy's formula

$$
f^{(n)}(z)=\frac{n !}{2 \pi i} \oint_{\gamma} \frac{f(t)}{(t-z)^{n+1}} d t
$$


holds for $n \in \mathbb{N}, z \in K$ and $f \in H(G)$. Observe that

$$
M:=\sup _{n \in \mathbb{N}} \frac{n ! \sup _{z \in K}\left|c_{n}(z)\right|}{d(K, \gamma)^{n}}
$$

is a finite constant because $A_{1}=0$. Choose $\delta=2 \pi \varepsilon d(K, \gamma) /(1+M$ length $(\gamma))$ and $L=\gamma$. If $n \in \mathbb{N}, f \in V(\delta, L)$ and $z \in K$ we have

$$
\begin{aligned}
\left|c_{n}(z) f^{(n)}(z)\right| & =\left|\frac{n ! c_{n}(z)}{2 \pi i} \oint_{\gamma} \frac{f(t)}{(t-z)^{n+1}} d t\right| \\
& \leq \frac{n ! \sup _{z \in K}\left|c_{n}(z)\right|}{2 \pi d(K, \gamma)^{n+1}} \cdot\|f\|_{\gamma} \cdot \operatorname{length}(\gamma) \\
& <\frac{M}{2 \pi d(K, \gamma)} \cdot \delta \cdot \operatorname{length}(\gamma)<\varepsilon .
\end{aligned}
$$

Consequently, (2) is satisfied and hence $\mathcal{F}$ is equicontinuous.

(b) It remains to show that if $A_{2}>0$ then $\mathcal{F}$ is not equicontinuous. Fix $a \in G$ and put $R:=d(a, \mathbb{C} \backslash G)=|a-b|$, where $b \in \mathbb{C} \backslash G$. Fix $r$ with $0<r<R$ and $R-r<A_{2}$. So $K:=\bar{B}(a, r)$ is a nonempty compact subset of $G$. Let $\varepsilon=1, L \in \mathcal{K}(G)$ and $\delta>0$. Let $m>0$ be so small that $m /|z-b|<\delta$ if $z \in L$. Then the function $f(z):=m /(z-b)$ belongs to $V(\delta, L)$ and

$$
\sup \left\{\left|c_{n}(z) f^{(n)}(z)\right|: z \in K\right\} \geq \frac{n ! \cdot m}{(R-r)^{n+1}} \inf _{z \in K}\left|c_{n}(z)\right| .
$$

But $R-r<A_{2}$, so that $R-r<\alpha_{2}(K)$ and

$$
\sup _{n \in \mathbb{N}}\left\{\frac{n ! \cdot m}{(R-r)^{n+1}} \inf _{z \in K}\left|c_{n}(z)\right|\right\}=+\infty .
$$

Therefore, by (3),

$$
\bigcup_{n \in \mathbb{N}}\left(c_{n}(\cdot) D^{n}\right)(V(\delta, L)) \not \subset V(1, K),
$$

which implies that $\mathcal{F}$ is not equicontinuous.

Let us see now what happens in the case $G=\mathbb{C}$.

TheOrem 2.4. Consider the following properties:

(a) There is an exhaustive sequence $\left\{K_{j}\right\}_{j \geq 1}$ in $\mathcal{K}(\mathbb{C})$ such that each $\alpha_{1}\left(K_{j}\right)$ is finite.

(b) The family $\mathcal{F}=\left\{c_{n}(\cdot) D^{n}: n \in \mathbb{N}\right\}$ of operators on $H(\mathbb{C})$ is equicontinuous.

(c) There is no $f \in H(\mathbb{C})$ such that the orbit $\left\{c_{n}(\cdot) f^{(n)}: n \in \mathbb{N}\right\}$ is dense in $H(\mathbb{C})$.

(d) $\left\{f \in H(\mathbb{C}):\left\{c_{n}(\cdot) f^{(n)}: n \in \mathbb{N}\right\}\right.$ is dense in $\left.H(\mathbb{C})\right\}$ is not residual. 
Then the following implications hold:

$$
(\mathrm{a}) \Rightarrow(\mathrm{b}) \Rightarrow(\mathrm{c}) \Rightarrow(\mathrm{d}) \text {. }
$$

Proof. It is trivial that $(\mathrm{b}) \Rightarrow(\mathrm{c}) \Rightarrow(\mathrm{d})$. To prove that $(\mathrm{a}) \Rightarrow(\mathrm{b})$ we fix $\varepsilon>0$ and $K \in \mathcal{K}(\mathbb{C})$. There is $j \in \mathbb{N}$ such that $K \subset K_{j}$ because $\left\{K_{j}\right\}_{j \geq 1}$ is exhaustive. But $\alpha_{1}\left(K_{j}\right)$ is finite, so there exists $n_{0} \in \mathbb{N}$ with

$$
\sup _{n \geq n_{0}}\left(n ! \sup _{z \in K_{j}}\left|c_{n}(z)\right|\right)^{1 / n}<\infty .
$$

We can now follow the proof of Theorem 2.3(1) step by step with the sole exception that we may choose the cycle $\gamma$ in such a way that

$$
\sup _{n \geq n_{0}}\left(n ! \sup _{z \in K_{j}}\left|c_{n}(z)\right|\right)^{1 / n} \leq d\left(K_{j}, \gamma\right) .
$$

Then, by choosing $L=\gamma$ and $\delta=2 \pi \varepsilon d\left(K_{j}, \gamma\right) / \operatorname{length}(\gamma)$, we obtain

$$
\bigcup_{n \geq n_{0}}\left(c_{n}(\cdot) D^{n}\right)(V(\delta, L)) \subset V\left(\varepsilon, K_{j}\right) \subset V(\varepsilon, K)
$$

and so we have (b) and the theorem is proved.

3. Antiderivative operators. We furnish a sufficient condition to get a certain property of $\left\{c_{n}(\cdot) I^{n}\right\}_{n=1}^{\infty}$-universality, which generalizes those given in [1] and [2]. It also turns out to be a sufficient condition for the equicontinuity of the family $\left\{c_{n}(\cdot) I^{n}: n \in \mathbb{N}\right\}$. Recall that in the definition of $I^{n}$ $(n \in \mathbb{N})$, a point $a \in G$ has been fixed.

Lemma 3.1. If $B_{1} \leq 1 / \Delta_{a}(G)$ and $f \in H(G)$, then $\left\{c_{n}(\cdot) I^{n} f\right\}_{n=1}^{\infty}$ converges to zero uniformly on compact subsets of $G$.

Proof. Let $z \in G$ be. From the definition of $\Delta_{a}(G)$, there exists a rectifiable arc $\gamma_{z} \subset G$ joining $a$ to $z$ such that $|z-t|<\Delta_{a}(G)$ for all $t \in \gamma_{z}$. Fix $K \in \mathcal{K}(G)$. Then we may choose a compact set $L$ and $\operatorname{arcs} \gamma_{z}$ in such a way that $\bigcup_{z \in K} \gamma_{z} \subset L \subset G$, $\sup _{z \in K} \operatorname{length}\left(\gamma_{z}\right)=S<\infty$ and $|z-t| \leq M=$ a constant $<\Delta_{a}(G)$ for all $z \in K$ and $t \in \gamma_{z}$. Fix $M_{1} \in\left(M, \Delta_{a}(G)\right)$. Since $B_{1} \leq 1 / \Delta_{a}(G)$, there is $n_{0} \in \mathbb{N}$ such that $\sup _{z \in K}\left|c_{n}(z)\right| /(n-1) ! \leq 1 / M_{1}^{n-1}$ whenever $n \geq n_{0}$. If $z \in K, n \geq n_{0}$ and $f \in H(G)$, we obtain

$$
\begin{aligned}
\left|c_{n}(z) I^{n} f(z)\right| & =\left|\frac{c_{n}(z)}{(n-1) !} \int_{\gamma_{z}}(z-t)^{n-1} f(t) d t\right| \\
& \leq \frac{\sup _{z \in K}\left|c_{n}(z)\right|}{(n-1) !} M^{n-1}\|f\|_{L} \operatorname{length}\left(\gamma_{z}\right) \\
& \leq S\|f\|_{L}\left(\frac{M}{M_{1}}\right)^{n-1} \rightarrow 0 \quad(n \rightarrow \infty) .
\end{aligned}
$$

Thus $\lim _{n \rightarrow \infty}\left\|c_{n}(\cdot) I^{n} f\right\|_{K}=0$, as required. 
Theorem 3.2. Assume that $B_{1} \leq 1 / \Delta_{a}(G)$. Then there exists a sequence $\left\{C_{n}\right\}_{n=1}^{\infty} \subset \mathbb{C}$ such that for every $f \in H(G)$ the sequence

$$
\left\{Q_{n}(z)=c_{n}(z) I^{n} f(z)+\sum_{j=0}^{n-1} \frac{C_{n-j}}{j !} z^{j}: n \in \mathbb{N}\right\}
$$

has the following properties:

(a) $\left\{Q_{n}(z): n \in \mathbb{N}\right\}$ is dense in $H(G)$.

(b) For every compact set $B \subset G$ with connected complement and every function $g$ which is continuous on $B$ and holomorphic in the interior of $B$, there is a subsequence of $\left\{Q_{n}(z): n \in \mathbb{N}\right\}$ converging to $g$ uniformly on $B$.

(c) For every Lebesgue-measurable set $E \subset G$ and every Lebesgue-measurable function $g: E \rightarrow \mathbb{C} \cup\{\infty\}$, there is a subsequence of $\left\{Q_{n}(z): n \in \mathbb{N}\right\}$ converging almost everywhere to $g$ on $E$.

Proof. Part (a) is a direct application of Lemma 3.1 and the above mentioned result of [8] to the domain $G$ and the function $\Phi \equiv 0$. Parts (b) and (c) are straightforward consequences of [8, Lemma 3].

Theorem 3.3. (a) If $B_{1} \leq 1 / \Delta_{a}(G)$, then the family $\mathcal{F}=\left\{c_{n}(\cdot) I^{n}\right.$ : $n \in \mathbb{N}\}$ of operators defined on $H(G)$ is equicontinuous.

(b) If $B_{2}>1 / d(a, \mathbb{C} \backslash G)$, then the family $\mathcal{F}$ is not equicontinuous.

Proof. (a) Fix $K \in \mathcal{K}(G)$ and $\varepsilon>0$. As in the proof of Lemma 3.1, find $L \in \mathcal{K}(G)$ and finite constants $M, M_{1}, S$ (with $M_{1} \in\left(M, \Delta_{a}(G)\right)$ ) such that

$$
\left|c_{n}(z) I^{n} f(z)\right| \leq S \cdot \delta\left(\frac{M}{M_{1}}\right)^{n-1} \leq S \cdot \delta=\varepsilon
$$

whenever $\delta=\varepsilon / S, n \geq n_{0}, z \in K$ and $\|f\|_{L} \leq \delta$. Therefore

$$
\bigcup_{n \geq n_{0}}\left(c_{n}(\cdot) I^{n}\right)(V(\delta, L)) \subset V(\varepsilon, K) .
$$

Thus the family $\left\{c_{n}(\cdot) I^{n}: n \geq n_{0}\right\}$ is equicontinuous, and hence so is $\mathcal{F}$.

(b) Fix two positive constants $\mu, \nu$ with $1 / d(a, \mathbb{C} \backslash G)<\mu<\nu<B_{2}$. Let $\delta>0$ and $L \in \mathcal{K}(G)$. Consider the compact set $K=\{z:|z-a|=1 / \mu\}$. Then there exists $m \in \mathbb{N}$ with $\delta(\nu / \mu)^{m}>1$ and $\inf _{z \in K}\left|c_{m}(z)\right|>m ! \nu^{m}$. The constant function $f \equiv \delta$ is obviously in $V(\delta+1, L)$ and for any $z \in K$ we have

$$
\begin{aligned}
\left|c_{m}(z) I^{m} f(z)\right| & =\left|\delta \cdot c_{m}(z) \cdot \frac{(z-a)^{m}}{m !}\right| \\
& \geq\left|\delta \cdot \inf _{z \in K}\right| c_{m}(z)\left|\cdot \frac{(1 / \mu)^{m}}{m !}\right| \geq \delta\left(\frac{\nu}{\mu}\right)^{m}>1,
\end{aligned}
$$

so $\left(c_{m}(\cdot) I^{n}\right)(V(\delta+1, L)) \not \subset V(1, K)$. Hence $\mathcal{F}$ is not equicontinuous. 
Corollary 3.4. Assume that $G=B(a, R)(R \in(0,+\infty])$ and consider the family of operators $\mathcal{F}=\left\{c_{n}(\cdot) I^{n}: n \in \mathbb{N}\right\}$. We have:

(a) If $B_{1} \leq 1 / R$, then $\mathcal{F}$ is equicontinuous on $H(G)$.

(b) If $\mathcal{F}$ is equicontinuous on $H(G)$, then $B_{2} \leq 1 / R$.

Acknowledgements. The author would like to thank L. BernalGonzález, K.-G. Grosse-Erdmann and the referee for helpful comments and suggestions.

\section{References}

[1] L. Bernal-González, Derivative and antiderivative operators and the size of complex domains, Ann. Polon. Math. 59 (1994), 267-274.

[2] C. Blair and L. A. Rubel, A triply universal entire function, Enseign. Math. 30 (1984), 269-274.

[3] S. M. Duios Ruis, Universal functions of the structure of the space of entire functions, Soviet Math. Dokl. 30 (1984), 713-716.

[4] R. M. Gethner and J. H. Shapiro, Universal vectors for operators on spaces of holomorphic functions, Proc. Amer. Math. Soc. 100 (1987), 281-288.

[5] G. Godefroy and J. H. Shapiro, Operators with dense, invariant, cyclic vector manifolds, J. Funct. Anal. 98 (1991), 229-269.

[6] K.-G. Grosse-Erdmann, Holomorphe Monster und universelle Funktionen, Mitt. Math. Sem. Giessen 176 (1987).

[7] J. Horváth, Topological Vector Spaces, Vol. 1, Addison-Wesley, Reading, 1966.

[8] W. Luh, Approximation by antiderivatives, Constr. Approx. 2 (1986), 179-187.

[9] G. R. MacLane, Sequences of derivatives and normal families, J. Anal. Math. 2 (1952), 72-87.

[10] J. C. Oxtoby, Measure and Category, 2nd ed., Springer, New York, 1980.

[11] W. Rudin, Real and Complex Analysis, 2nd ed., Tata McGraw-Hill, Faridabad, 1974.

Departamento de Análisis Matemático

Facultad de Matemáticas, apdo. 1160

Avenida de Reina Mercedes

41080 Sevilla, Spain

E-mail: mccm@cica.es 